\title{
Enhanced electrophoretic DNA separation in photonic crystal fiber
}

\author{
Yi Sun ${ }^{l}$, Nam-Trung Nguyen ${ }^{2}$ and Yien Chian Kwok ${ }^{l}$ \\ 'National Institute of Education, Nanyang Technological University, 1 Nanyang \\ Walk, Singapore, 637616, Singapore \\ ${ }^{2}$ School of Mechanical and Aerospace Engineering, Nanyang Technological \\ University, 50 Nanyang Avenue, Singapore,639798, Singapore \\ Corresponding author: Dr. Yien Chian Kwok, National Institute of Education, \\ Nanyang Technological University, 1 Nanyang Walk, Singapore. \\ E-mail: yienchian.kwok@nie.edu.sg
}

\begin{abstract}
Joule heating generated by the electrical current in capillary electrophoresis leads to a temperature gradient along the separation channel and consequently affects the separation quality. We describe a method of reducing Joule heating effect by incorporating photonic crystal fiber into a micro capillary electrophoresis chip. The photonic crystal fiber consists of a bundle of extremely narrow hollow channels, which ideally work as separation columns. Electrophoretic separation of DNA fragments was simultaneously but independently carried out in 54 narrow capillaries with diameter of $3.7 \mu \mathrm{m}$ each. The capillary bundle offers more efficient heat dissipation owing to high surface-to-volume ratio . Under the same electric field strength, notable improvement in resolution was obtained in the capillary bundle chip.
\end{abstract}

\section{Keywords}

Joule heating / Capillary bundle / Photonic crystal fiber / Microchip capillary electrophoresis / DNA separation

\section{Introduction}

Capillary electrophoresis (CE) usually involves high applied voltage. Owing to the presence of electrical potential gradient and electrical current in electrokinetic flow, Joule heating is an inevitable phenomenon and leads to an increase in the overall temperature and temperature gradients in the transverse and longitudinal directions inside the capillaries [1]. Excess temperature elevations may cause bubble formation, denaturation of biological samples, and even breakdown of the chip systems [2]. Large temperature gradients cause band broadening and dispersion which leads to inefficient and low-quality separation [3]. It was assumed that in microchannels, the large channel surface-to-volume ratio (SVR) would reduce the Joule heat and increase the cooling rate through the channel wall [4]. However, many research works in the past showed that Joule heating still affect electrophoretic separation in microchannels. Swinney and Bornhop [5] observed a 7\% reduction in separation efficiency for tris(hydroxymethyl)aminomethane/boric acid buffer under an applied field of 475 $\mathrm{V} / \mathrm{cm}$ in comparison to $240 \mathrm{~V} / \mathrm{cm}$. Chen and Chen [6] reported that plate number of DNA fragment started to decrease when the electric field strength was greater than $300 \mathrm{~V} / \mathrm{cm}$. 
As Joule heating sets an upper limit for the use of high electrical voltages, effective dissipation of the Joule heat is critical for reproducible and efficient separations in electrokinetically driven systems. One simple solution to diminish Joule heat effect is to decrease the cross-section of the microchannel [7]. The heating rate generated by the electric field is a square function of the applied voltage. The smaller the channel cross-section, the larger is the electrical resistance. Consequently, less electrical current and less heat are generated for a given voltage. In addition, the heat can be dissipated more quickly from a microchannel with smaller cross-sectional area because of the favorable SVR.

Previously, we presented a novel approach of performing microchip CE in a bundle of extremely narrow channels by using photonic crystal fiber (PCF) as separation column [8]. PCF consists of a bundle of narrow capillaries, each capillary with a diameter on the order of few micrometers. Capillaries with such small cross section can sustain high electric field up to $1,000 \mathrm{~V} / \mathrm{cm}$ and give better separations owing to enhanced heat dissipation and smaller temperature differences across the cross section. PCFs are commercially available at a reasonable cost, and making separation devices with PCF would not need microfabrication facilities. The fluorescence signals were collected from all the capillaries, thus the available detection volume of a capillary bundle was comparable with that of a normal microchip. In the work reported here, the capillary bundle microchip was employed for electrophoretic separation of DNA fragments. The separation was simultaneously but independently carried out in 54 narrow channels. More efficient heat dissipation, thus enhanced separation was achieved on the capillary bundle chip.

\section{Experimental}

\section{Materials and fabrication}

The concept of making PCFs was based on the same concept used for making conventional solid-core/solid-cladding fibers. First, a bundle perform consisting of many small hollow capillary with a typical diameter on the order of centimeters was prepared. The preform was then heated and drawn down to a much smaller diameter. During this process, the shape of the cross section of the preform is maintained, but the diameters of the hollow capillaries reduce to a few microns. Most PCFs are fabricated in silica glass [9]. Instead of guiding light, we used PCF as a bundle of microchannels for CE separation. Figure 1a shows the scanning electron microscopy images of the cross section of the capillary bundle used in our experiments. The PCF (ESF-12-01, Blaze Photonics, UK) has an outer diameter of $125 \mu \mathrm{m}$. There are 54 capillaries with a channel diameter of $3.7 \mu \mathrm{m}$ each, giving a total cross-section area of $580.3 \mu^{2}$. The PCF was cut into small pieces of $7 \mathrm{~cm}$ which work as the separation column in the later CE microdevice as shown in Fig 1b. The fabrication process of the capillary bundle chip was described before[8]. Briefly, a microchannel network was engraved in poly(methyl methacrylate) (PMMA) substrate by $\mathrm{CO}_{2}$ laser ablation [10]. The network consists of a $100-\mu \mathrm{m}$-wide and 30- $\mu \mathrm{m}$-deep T-shaped injector for electrokinetic sample injection and a $200-\mu \mathrm{m}$-wide and $150-\mu \mathrm{m}$-deep straight channel for holding the PCF. After the 7-cm-long PCF separation column had been inserted, polydimethylsiloxane (Best Chemicals, Singapore) was used to fill the gap between the PCF and the channel engraved by the laser to make sure that the outer areas of the PCF was properly sealed. The whole substrate was baked at $60{ }^{\circ} \mathrm{C}$ on hotplate for 30 
min, and the device was then sealed by a layer of adhesive tape (Adhesives Research, Glen Rock, PA, USA).

The performance of the capillary bundle was compared to a commercially available glass microchip (X8050, Micronit Microfluidic, The Netherland). The chip has a microchannel with $50 \mu \mathrm{m} \times 20 \mu \mathrm{m}$ cross-section. Considering the curved side walls caused by isotropic etching, the cross-section area of the glass microchannel was approximately $600 \mu \mathrm{m}^{2}$, which was comparable to the total cross-sectional area of the 54 capillaries of the capillary bundle. Joule's Law states that the amount of heat generated in an electrical system can be calculated by

$$
J(t)=i V t=\frac{V^{2} t}{R}=\frac{V^{2} \sigma A t}{L},
$$

where $J$ is the heat generated (joules) as a function of time $t$, when a potential drop is applied across a capillary of resistance, $R$, with area of cross-section $A$ and length $L$ [10]. Since both chips have similar cross-section area, the same amount of Joule heat was generated during electrophoretic separation process. However, according to the equation for SVR of a microchannel with a unit length,

$$
\mathrm{r}_{\mathrm{SV}}=\frac{\mathrm{S}}{\mathrm{V}}=\frac{\text { perimeter of cross section }}{\text { area of cross section }}
$$

the SVR of the capillary bundle was $1.1 \mu \mathrm{m}^{-1}, 8$ times larger than $0.15 \mu \mathrm{m}^{-1}$ for the glass microchip.

\section{Chemicals}

The separation running buffer consisted of $1.5 \%$ hydroxypropylcellulose (molecular weight 100,000, Sigma, MO, USA) $80 \mathrm{mM}$ 2-morpholinoethanesulfonic acid/40 mM tris(hydroxymethyl)aminoethane (Sigma, MO, USA). Intercalating dye YOPRO-1 (Invitrogen, USA) was added to a concentration of $10 \mu \mathrm{M}$. The DNA sizing ladder, ФX174-Hae III double-stranded DNA digest (Invitrogen, USA), was dissolved in autoclaved deionized water to a final concentration of $5 \mu \mathrm{g} / \mathrm{mL}$.

\section{CE separation}

CE separation was carried out using both the capillary bundle and the glass microchip with an inverted confocal microscope (TCS SP2, Leica, Germany) as the detection apparatus [11]. The voltages for the four reservoirs were generated by a high voltage power supply (MCP 468, CE Resources, Singapore). For DNA separation, an electric field of $500 \mathrm{~V} / \mathrm{cm}$ was applied across the buffer and the buffer waste reservoirs.

\section{Results and discussion}

As Joule heating is dissipated via the channel surface, the capillary bundle microchip can dissipate Joule heat more effectively and thus be able to sustain much higher electric field strengths. Previously, the capability of the capillary bundle chip 
to dissipate the Joule heat was investigated by monitoring the electrical current as a function of the applied field strength [8]. The result showed that the current in the glass microchip was generally higher than that in capillary bundle. From the Ohm's plots, the glass could only bear an electric field lower than $700 \mathrm{~V} / \mathrm{cm}$; while the capillary bundle microchip could withstand an electric field up to $1,100 \mathrm{~V} / \mathrm{cm}$. Therefore, by introducing PCF, we greatly increased the effective range of separation electric field strength.

The flow profile of DNA sample in PCF during separation was investigated by taking fluorescent images at the point $1 \mathrm{~cm}$ from the buffer waste reservoir. Figure 2 shows that DNA fragments of the same size moved at the same speed in all the microchannels, and there was no variation in migration velocities which would otherwise lead to broader bands and reduced resolution. In the capillary bundle, the DNA sample was well confined in the capillaries and there was no leakage to the PMMA microchannel, indicating that the PCF was completely sealed by polydimethylsiloxane and electrophoretic separation was independently carried out in each narrow capillary.

Figures 3 shows the electropherograms obtained in the glass bundle chip and in the glass microchip. The resolutions of the DNA fragments obtained in the capillary bundle chip and in the glass microchip are summarized in Table 1. As seen from the results, for the glass microchip, fragments $271 / 281$ as well as $1,078 / 1,353$ were unable to be separated. Moreover, the migration times for each fragment were relatively shorter than the migration time in the capillary bundle chip. This provided evidence of inefficient heat dissipation in the glass microchip as higher temperature would result in a corresponding decrease in the viscosity of the hydroxypropylcellulose matrix and thus faster migration velocities of the DNA fragments. The lower viscosity directly led to deformed sieve size and reduced resolving power of the sieving matrix. Since the quality of DNA separation largely depends on the matrix, the decreased viscosity of the separation medium caused by Joule heat adversely affected the separation efficiency and the analysis resolution in the glass microchip. In contrast, under the same electric field strength, notable improvement in resolution was obtained in the capillary bundle chip. This could be attributed to the relatively larger SVR of the capillary bundle and the better controlled Joule heating and temperature gradients. Consequently, viscosity as well as resolving power of sieving matrix were retained at higher voltage. The enhanced resolution obtained using the capillary bundle would facilitate its wide applicability for DNA separation in various fields.

\section{Conclusion}

In conclusion, improved electrophoretic separation of DNA fragments was demonstrated in a bundle of extremely narrow channels by using PCF as separation column. Compared to a normal glass microchip with similar cross-section area, the capillary bundle has a much larger SVR; hence higher resolution was obtained owing to efficient heat dissipation. As the PCF is commercially available and the fabrication process to encapsulate PCF to PMMA substrate is simple, this novel concept could be widely applied to chip-based DNA separation for enhanced performance. 


\section{References}

[1] Cetin, B, Li, DQ(2008) Electrophoresis 29:994-1005.

[2] Xuan, XC (2008) Electrophoresis 298:33-43.

[3] Ghosal, S (2004) Electrophoresis 25:214-228.

[4] Petersen, NJ, Nikolajsen, RPH, Mogensen KB, Kutter, JP(2004), Electrophoresis 25:253-269.

[5] Swinney, K, Bornhop DJ(2002), Electrophoresis 23:613-620.

[6] Chen, YH, Chen SH(2000), Electrophoresis 21:165-170.

[7] Kuhn R, Kuhn, SH(1998) Capillary electrophoresis: principles and practice. Springer,Berlin

[8] Sun, Y, Kwok, YC, Nguyen NT(2007), Electrophoresis 4765-4768.

[9] Chillcce, EF, Cordeiro CMB, Barbosa LC, Cruz CHB(2006) J. NonCrystalline Solids 2006, 352, 3423-3428.

[10] Rathore AS(2004), J Chromatogr. A 1037:431-443.

[11] Sun, Y., Kwok, YC, Nguyen, NT(2006) J Micromech. Microeng. 16:16811688 . 


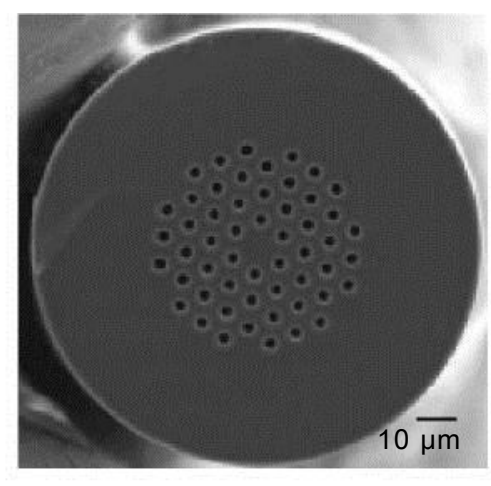

(a)

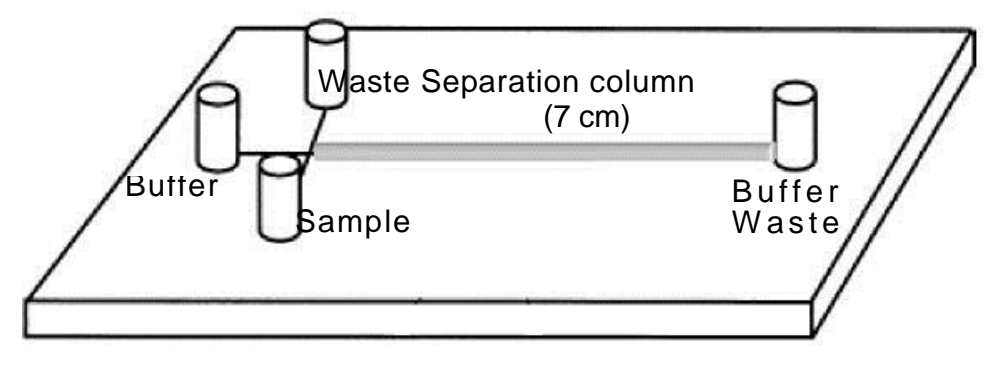

(c)

Figure 1. a Scanning electron microscopy image of the cross-section of the capillary bundle. b Layout of the capillary electrophoresis microchip with capillary bundle. 


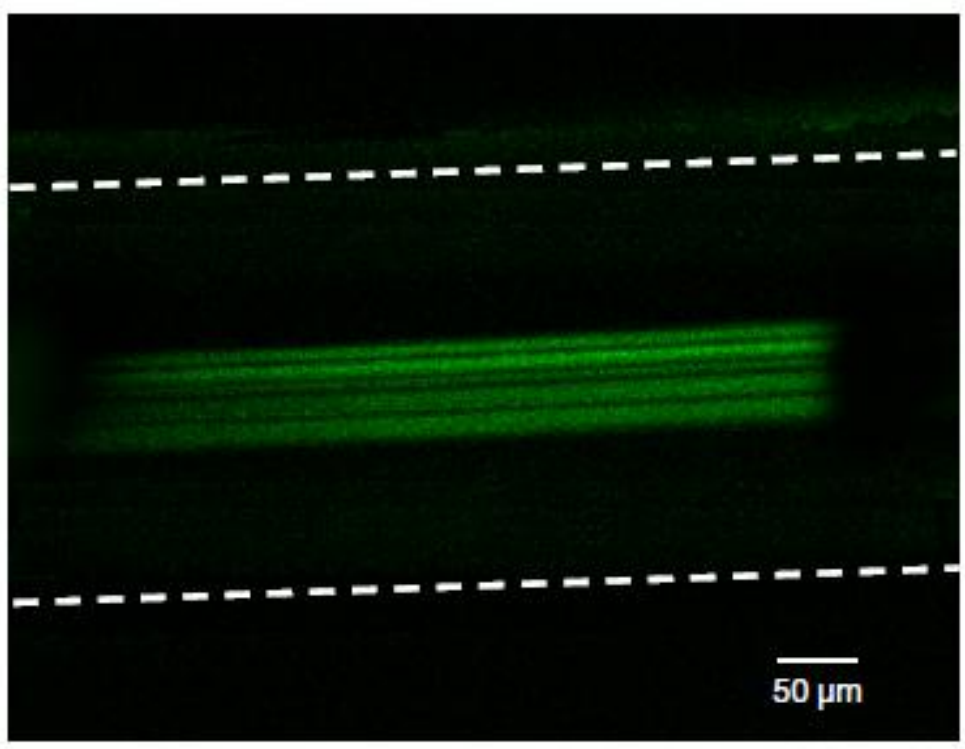

Figure 2. Flow profile of DNA fragment in photonic crystal fiber during separation. Fluorescent images were taken at the detection point $1 \mathrm{~cm}$ from the buffer waste reservoir. 


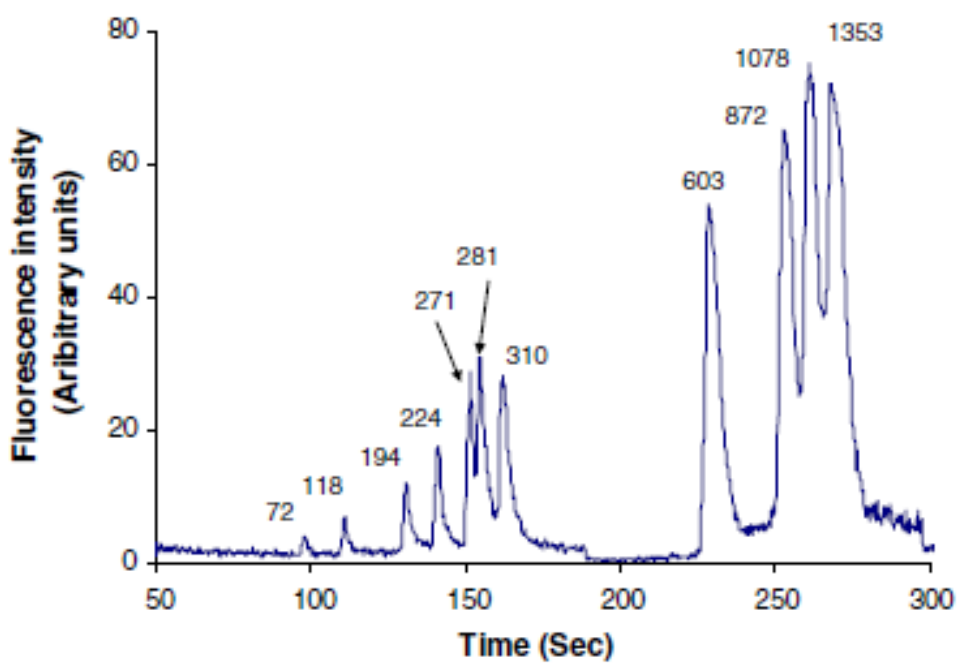

(a)

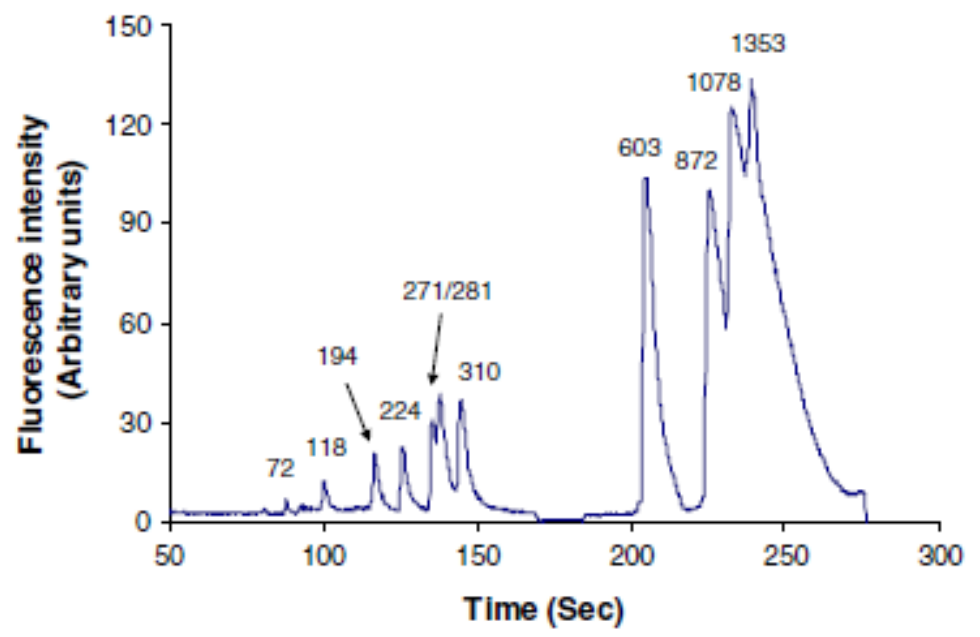

(b)

Figure 3. Electropherograms of $5 \mu \mathrm{g} / \mathrm{mL}$ ФX174-HaeIII double-stranded DNA digest in $80 \mathrm{mM}$ 2-morpholinoethanesulfonic acid/40mM tirs(hydroxymethyl)aminomethane buffer with $1.5 \%$ hydroxypropylcellulose on a the capillary bundle and $b$ the glass microchip under an applied electrical field of $500 \mathrm{~V} / \mathrm{cm}$ with separation length of 6 $\mathrm{cm}$. The results were repeated three times. The standard deviations are less than 0.5 and $5 \%$ for migration time and half peak width, respectively. 


\begin{tabular}{lll}
\hline DNA fragment (bp) & \multicolumn{2}{l}{ Resolution } \\
\cline { 2 - 3 } & Glass microchip & Capillary bundle chip \\
\hline 72 & - & - \\
118 & 2.2 & 3.2 \\
194 & 1.1 & 5.8 \\
224 & 2.0 & 2.7 \\
271 & 1.9 & 2.8 \\
281 & 0.4 & 0.8 \\
310 & 0.9 & 1.4 \\
603 & 7.3 & 8.7 \\
872 & 2.1 & 2.7 \\
1,078 & 0.6 & 0.9 \\
1,353 & 0.3 & 0.6 \\
\hline
\end{tabular}

Table 1 Resolutions of the DNA fragments obtained in the capillary bundle and the glass microchip 